Cahiers de recherche sociologique

RECHERCHE

SOCIOLOGIQUE

\title{
Entre résistance et commercialisation : à la recherche du renouveau politique
}

Présentation

\section{Marie Nathalie LeBlanc}

Numéro 49, hiver 2010

Dilemmes hip-hop

URI : https://id.erudit.org/iderudit/1001409ar

DOI : https://doi.org/10.7202/1001409ar

Aller au sommaire du numéro

Éditeur(s)

Athéna éditions

ISSN

0831-1048 (imprimé)

1923-5771 (numérique)

Découvrir la revue

Citer ce document

LeBlanc, M. N. (2010). Entre résistance et commercialisation : à la recherche du renouveau politique : présentation. Cahiers de recherche sociologique, (49),

5-15. https://doi.org/10.7202/1001409ar d'utilisation que vous pouvez consulter en ligne.

https://apropos.erudit.org/fr/usagers/politique-dutilisation/ 


\title{
Entre résistance et commercialisation: à la recherche du renouveau politique Présentation
}

\author{
Marie Nathalie LeBlanc
}

L

E TERRAIN DE LA CUlTURE populaire nord-américaine s'est redéfini grâce à l'essor et à la mondialisation du hip-hop à la fin des années 1980. De nos jours, la culture hip-hop continue d'évoluer au sein du marché mondial de la musique populaire. Les premiers artistes hip-hop aux États-Unis témoignaient de leurs conditions de vie à travers divers modes d'expression - musicaux avec les rappeurs et les DJ qui pratiquent le platinisme, le turntabalism ou le scratching; corporels par le breakdancing des b-boys et b-girls ou graphiques chez les graffitistes ou les bombeurs. Au-delà de chacune de ces formes artistiques, les artistes et leurs auditoires considèrent le hip-hop comme un mode de vie associé à l'appropriation des espaces urbains ${ }^{1}$; de là découle la notion de «culture hip-hop».

Au-delà de son expressivité, la culture hip-hop est peut-être l'expérience la plus réussie en matière de médiatisation de communautés dites marginales et de mondialisation culturelle. Que ses racines soient retracées dans la tradition griotique d'Afrique de l'Ouest, dans les chansons de travail des esclaves du Delta du Mississippi aux États-Unis ou encore dans la musique dancehall

1. Hugues Bazin, La culture hip-hop, Paris, Desclée de Brouwer, 1995. 
des Caraibes², la naissance de la musique hip-hop dans les années 1970 est généralement associée au DJ d'origine jamaïcaine Clive Campbell. En effet, DJ Kool Herc, de son nom d'artiste, organisait des fêtes dans le Bronx newyorkais au 1520, Sedgwick Avenue. En utilisant deux platines de mixages jointes pour enchevêtrer des percussions rythmées avec de la musique funk, il développe la technique électronique du «rythme cassé » (breakbeat), caractérisé par des rythmes binaires très syncopés et qui donnera lieu à l'échantillonnage (sampling), caractéristique principale qui démarquera le rap des autres styles musicaux. Les invités du 1520, Sedgwick Avenue étaient libres de prendre le micro et de rapper sur cette musique, afin de mettre en scène leur habileté verbale et d'exprimer leur créativité spontanément. Ces soirées ont été déterminantes pour le son et le sens de la communauté qui ont forgé les pionniers du hip-hop américain, notamment les rappeurs Grandmaster Flash, Afrika Bambaata et The Sugarhill Gang.

À l'origine, les thèmes du rap, qui reste l'expression la plus commercialisée de la culture hip-hop, s'inscrivaient dans les dynamiques sociales, politiques et raciales du milieu urbain new-yorkais ${ }^{3}$. Des groupes engagés, tels que Public Enemy, ont documenté à travers leur musique les formes contemporaines de l'inégalité aux États-Unis ${ }^{4}$ tout en publicisant la centralité des dynamiques de racialisation au sein de cette société ${ }^{5}$. C'est ainsi que les premiers artistes du hip-hop américain développèrent une culture dite d'opposition $^{6}$ ou de résistance en référence à la pauvreté et à la discrimination raciale qui sévissaient dans les ghettos noirs ${ }^{7}$. Tout en dénonçant l'expérience vécue quotidienne de la discrimination raciale aux États-Unis, cette culture s'est construite à l'intersection de l'héritage de l'expérience de l'esclavagisme et de la réalité quotidienne de la culture noire transnationale ${ }^{8}$. Il s'agissait donc d'un mouvement artistique de portée sociopolitique mis en scène à travers une culture de la fête (ou block parties). Pour une génération alors privée de son patrimoine culturel, le hip-hop représentait une forme d'expression révolutionnaire. Dès son émergence, la musique hip-hop et son message de résistance aux diverses formes de la domination socio-économique ont attiré

2. Halifu Osumare, The Africanist Aesthetic in Global Hip-Hop: Power Moves, New York, Palgrave MacMillan, 2007.

3. H. Bazin, op. cit.

4. Russell A. Potters, Spectacular Vernaculars: Hip-Hop and the Politics of Postmodernism, Albany, State University of New York Press, 1995, p. 13.

5. Theresa A. Martinez, «Popular Culture As Oppositiona; Culture: Rap as Resistance», Sociological Perspectives, vol. 10, n², 1997, p. 268.

6. Ibid., p. 265.

7. Michael E. Dyson, «Performance, Protest, and Prophecy in the Culture of Hip-Hop», Black Sacred Music: A Journal of Theomusicology, vol. 1, p. 17.

8. Paul Gilroy, L'Atlantique noir. Modernité et double conscience, Paris, Éditions Kargo, 2003. 
un public diversifié en raison de sa capacité d'incarner la réalité des groupes marginalisés ou minorisés.

Conçu par les Américains d'origine africaine, latino et antillaise ${ }^{9}$, ce type de représentation du quotidien transcende aujourd'hui les frontières linguistiques, culturelles, raciales et nationales. La culture hip-hop est en effet largement consommée et diffusée à l'échelle mondiale. La littérature existante sur ce phénomène révèle que ce genre populaire reflète non seulement l'idéologie afro-américaine dans laquelle il puise ses racines, mais aussi le contexte socio-économique et culturel au sein duquel il se façonne.

À la fin des années 1980, le centre névralgique de la culture hip-hop s'est déplacé du Bronx à Compton, banlieue pauvre de Los Angeles, où les artistes, tels que Eazy-E, Too Short, Ice T et N.W.A., parmi de nombreux autres, ont fait connaitre le gagsta rap. L'émergence de ce style de rap marque symboliquement le passage à une entreprise de commercialisation et il est, encore aujourd'hui, le plus diffusé des styles musicaux provenant des États-Unis à l'échelle mondiale ${ }^{10}$. En raison de son articulation, symbolique ou réelle, avec la violence, le sexisme, la magouille, la criminalité, la drogue, le proxénétisme des rues de Compton, le gangsta rap est aussi vu comme l'apogée du matérialisme bling-bling, une des expressions culturelles du consumérisme américain, et l'expression la plus caricaturale de la violence associée à la jeunesse noire, souvent contrasté avec le rap engagé et militant de formations comme Public Enemy et Digable Planet.

En raison des dynamiques économiques de la mondialisation, des innovations technologiques permettant la microproduction et du succès commercial du gansta rap américain, le phénomène prit une ampleur considérable au cours des deux dernières décennies ${ }^{11}$. La culture hip-hop évolue maintenant au sein du marché global de la musique commerciale. On peut donc dire que les premiers artistes hip-hop ont impulsé un mouvement social qui, à l'heure actuelle, se pense comme l'une des principales sources de la culture

9. Pour les études classiques et pionnières de la culture hip-hop des États-Unis, voir Nelson George, Hip-hop America, New York, Penguin Books, 1999; Tricia Rose, Black Noise: Rap Music and Black Culture in Contemporary America, Middleton, Wesleyan University Press, 1994.

10. Voir G. Alvarez, "Gansta Rap in the '90s», dans A. Light (dir.), The Vibe History of Hip Hop, New York, Random House, 1999, p. 285-295.

11. Voir Tony Mitchell, «Doin' Damage in my Native Language: The Use of 'Resistance Vernaculars' in Hip Hop in Europe and AotearoalNew Zealand», dans Sheila Whiteley, Andy Bennet et Stan Hawkins (dir.), Music, Space and Place: Popular Music and Cultural Identity, Hants et Burlington, Aldershot et Ashgate, 2004; Halifu Osumare, The Africanist Aesthetic in Global Hip-Hop: Power Moves, New York, Palgrave MacMillan, 2007. 
populaire des jeunes à travers le monde en lien avec diverses expériences de marginalisation $^{12}$.

En dépit de son caractère apparemment universaliste, la culture hiphop se construit en réalité au niveau local, en fonction des enjeux sociaux, politiques, économiques et culturels qui caractérisent chaque espace. Andy Bennett, sociologue ayant travaillé sur le rap au nord-est de l'Angleterre, constate que le hip-hop fournit aux jeunes Anglais blancs d'origine ouvrière une plateforme pour l'action sociale ${ }^{13}$. En France, ce sont les citoyens d'origine immigrée, principalement maghrébine, qui ont introduit le rap en l'utilisant comme une tribune d'expression de leur mal-être urbain, mal-être souvent relié par ailleurs à l'expérience de l'immigration et à l'exclusion sociale ${ }^{14}$. En Allemagne, le hip-hop permet aux jeunes Turcs de soulever les questions de racisme vécu et les problèmes liés à la citoyenneté ${ }^{15}$.

Du Japon à l'Afrique, de l'Asie à l'Europe de l'Est, de jeunes artistes s'affairent à mettre en scène leur milieu de vie respectif en produisant des réponses locales, adaptées à leurs propres interactions avec la culture populaire globale. Toutefois, les références historiques et identitaires des AfroAméricains n'en continuent pas moins d'informer et d'inspirer les formes localisées du hip-hop. Tel que le suggère Tricia Rose,

the drawing power of rap is precisely its musical and narrative commitment to black youth and cultural resistance, and nothing in rap's commercial position and crosscultural appeal contradicts this fact ${ }^{16}$.

De fait, le symbole fort de la «négritude» évoquée par le mouvement hip-hop est devenu un produit identitaire et commercial qui traverse les barrières géographique, linguistique, raciale, nationale et religieuse, et qui est diffusé à grande échelle à travers le monde ${ }^{17}$. Qu'on le nomme favela funk,

12. Voir N. George, op. cit.; A. Herman et al., "Mapping the Beat: Space of Noise and Places of Music», dans A. Herman et al. (dir.), Mapping the Beat: Popular Music and Contemporary Theory, Oxford Blackwell Publishers, 1998, p. 3-29; D. C. M. Hesmondhalgh, «Urban Breakbeat Culture», dans T. Mitchell (dir.), Global Noise: Rap and Hip Hop Outside of the USA, Middletown, Wesleyan University Press, 2001, p. 86-110; T. Mitchell (dir.), ibid.; H. Osuware, «Beat Streets in the Global Hood: Connective Marginalities of the Hip Hop Globe, Journal of American and Comparative Cultures, vol. 24, p. $171-181$.

13. Andy Bennett, «Rappin' On the Tyne: White Hip-hop Culture in Northeast England: An Ethnographic Study», The Sociological Review, vol. 47, n 1, 1999, p. 1-24.

14. H. Bazin, op. cit.; G. Lapassade et P. Rousselot, Le rap ou la fureur de dire, Paris, Loris Talmart, 2000.

15. A. Kaya, Sicher in Kreuzberg. Constructing Dispora: Turkish Hip Hop Youth in Berlin, Piscataway, Transaction Publisher, 2001.

16. Traduction libre de l'auteure: «le pouvoir d'attirance du rap se situe justement au niveau de son engagement musical et narratif envers la jeunesse noire et la résistance culturelle, et rien dans la position commerciale et dans son attrait interculturel ne contredit ce fait». Tricia Rose, Black Noise..., op. cit., p. 19.

17. Voir P. Gilroy, op. cit. 
dancehall, Miami bass, hip-house, grime, kwaito, sleaze, freestyle, desi, reaggaton ou hip-life, le hip-hop reste une musique de la rue polyglotte qui reformule sans cesse des réinterprétations populaires à l'image de la géographie de son appropriation.

Alors qu'initialement le hip-hop était de forme descriptive et qu'il traduisait la quête de reconnaissance de ceux qui ne disposaient d'aucun autre moyen de se faire entendre, cette culture est aujourd'hui confrontée aux dynamiques de la commercialisation, à la fois à l'échelle internationale, et à celle des politiques et des industries culturelles dans divers contextes nationaux. Il en ressort que les acteurs se mesurent aujourd'hui aux dilemmes de l'«authenticité» dans le cadre de la société de consommation. En d'autres termes, comment le hip-hop peut-il rester un véhicule de dénonciation et d'affirmation culturelle tout en étant partie prenante des dynamiques capitalistes de la marchandisation?

De fait, le «Hip-hop est mort» est l'eulogie du jour depuis près de 30 ans, depuis la mise en place de la domination du gansta rap dans la culture musicale commerciale américaine. Toutefois, au-delà de la tension inhérente entre les soi-disant expressions commerciales et politiques du hip-hop, Tricia Rose $^{18}$ resitue les dilemmes de la culture hip-hop contemporaine en termes d'une «guerre culturelle» qui opposent ses détracteurs et ses défenseurs, guerre d'interprétation dans laquelle les détracteurs maintiennent que cette culture expressive glorifie, encourage et cause la violence, tandis que ses supporteurs soulignent la nécessité de maintenir son authenticité, son attachement aux expériences de la rue et de la quotidienneté de l'oppression, captée par la notion populaire de keeping it real ("gardez ça vrai»). Ces derniers mettent en valeur le rôle expressif et réflexif du hip-hop dans le contexte des ghettos noirs américains. Rose conclut son ouvrage par une exhortation renouvelée à la revitalisation des ressources créatrices du hip-hop comme paradigme de la résistance des jeunes et du changement social dans l'ère des droits civils aux États-Unis. À son avis, l'esprit créateur du hip-hop se situe en marge de la lecture dichotomique entre le rap politiquement conscientisé et sa seule alternative les "ganstas, pimps and hoes ${ }^{19}$ ».

Ce constat ouvre le débat sur les dilemmes contemporains de la culture hip-hop de l'implication de plus en plus généralisée de ces acteurs dans une forme d'activisme. Harry Allen, intellectuel, activiste et journaliste américain, parlera d'un nouvel «activisme hip-hop» pour qualifier l'engagement de

\footnotetext{
18. Tricia Rose, The Hip Hop Wars: What We Talk About When We Talk About Hip Hop - and Why It Matters, New York, Basic Books, 2008.

19. Ibid., p. 243.
} 
la génération post-baby boomers aux États-Unis. Rose mentionne, entre autres, les initiatives de HELP (Hip-hop Educational Literacy Program) qui utilise le hip-hop dans des programmes d'éducation populaire ${ }^{20}$, Hip-Hop 4 Humanities $^{21}$ qui organise des camps d'été pour la jeunesse défavorisée noire, de concert avec le Georgia State University, ou encore HOTGIRLS, un programme d'éducation sexuelle pour les jeunes filles noires ${ }^{22}$. Pour sa part, Jenny Mbaye parlera plutôt d' «activisme musical» dans son étude du mouvement AURA en Afrique de l'Ouest ${ }^{23}$. Dans ce contexte, la notion d'activisme implique une interpénétrabilité entre les expressions artistiques et les actions des acteurs en dehors du cadre musical ou artistique.

Cet appel au renouveau culturel, mais aussi à la conscientisation politique, a impulsé le regain du champ de connaissance de cette culture et de la culture populaire mondialisée, de façon plus générale. De plus en plus, les chercheurs tendent à aborder les dilemmes contemporains de la culture hiphop mondialisée en les inscrivant en dehors de la dichotomie entre culture commerciale et culture de la revendication. Les études récentes favorisent aussi un regard transculturel quant à la lecture du dilemme nord-américain opposant résistance et commercialisation, en proposant de les relire à la lumière d'autres contextes de l'appropriation des expressions et des idiomes culturels du hip-hop.

Les sept articles présentés dans ce numéro spécial harponnent certaines des tendances d'analyse qui se dessinent dans ce nouveau champ de connaissance. Tandis que les articles de Gabriella Djerrahian, Abaher El Sakka et Abdoulaye Niang situent l'analyse du politique en termes des dynamiques entre les enjeux locaux de la reconnaissance et les forces de la mondialisation, ceux de Alexandrine Boudreault-Fournier, Hugues Bazin, Naïm Bornaz et Mehdi Slimani, Jenny Mbaye et Myriam Laabidi mettent l'accent sur les interstices et l'interpénétration des dynamiques de la domination marchande et de la résistance politique. Ces articles montrent, dans une perspective conjoncturelle qui met en lumière des dynamiques particulières aux différentes géographies, que la lecture linéaire de l'évolution de cette culture, à partir d'un temps original mythique centré sur la dénonciation des inégalités sociales et économiques à la mise à mort du hip-hop par sa marchandisation, masque la complexité des dilemmes auxquels les acteurs contemporains de la culture hip-hop mondialisée font face.

\footnotetext{
20. www.edlyrics.com

21. www.hh4h.com

22. www.helpingourteengirls.org

23. Voir plus loin dans ce numéro.
} 
Les articles reflètent aussi l'érudition d'une génération de jeunes chercheurs qui publient en langue française dans un champ de connaissance majoritairement dominé par les publications en anglais et par les auteurs américains. Aux États-Unis, l'étude de la culture hip-hop constitue un champ scientifique en soi avec des revues spécialisées et des programmes d'études et ses propres débats et postures théoriques. Sans nier l'importance de comprendre les dynamiques de la revendication identitaire et de la dénonciation des relations de domination dans les lieux centraux des industries culturelles, principalement aux États-Unis, nous avons choisi de focaliser sur des lieux plus périphériques aux dynamiques de la commercialisation musicale et à la naissance de la culture hip-hop transnationale, c'est-à-dire Cuba, le Sénégal, l'Afrique de l'Ouest, la Palestine, Israël et le Québec. Le regard porté sur ces autres espaces permet de mettre en lumière la complexité de l'articulation entre les dynamiques de la marchandisation et de la résistance, tout autant que la pertinence régionale et les expressions locales divergentes de ces dynamiques. En d'autres termes, la perspective transculturelle adoptée dans ce numéro spécial pousse à repenser les termes et les solutions à envisager par les acteurs sociaux.

Gabriella Djerrahian et Abaher El Sakka examinent, par l'entremise du rap palestinien et de celui des Israéliens d'origine éthiopienne, les dynamiques de la racialisation des rapports sociaux et de la discrimination en Israël chez des jeunes et des artistes qui cherchent à faire reconnaitre leur art. El Sakka analyse les symboles qui proviennent de divers registres et que mobilisent les artistes pour susciter l'attention de leurs auditeurs potentiels. Ces symboles varient de l'emblème politique du keffieh à des jeux de mots qui se moquent des gouvernements du Fatha et du Hamas ou qui ironisent sur le terme «terroriste", sans oublier de mobiliser certains des outils privilégiés de la marchandisation de la culture hip-hop dont Internet et le projet de lancer une Star Academy du rap et du hip-hop palestiniens.

Djerrahian, pour sa part, déconstruit la logique qui allie le hip-hop à la conscience racialisée chez les jeunes Israéliens d'origine éthiopienne, à savoir comment la référence globalisée de la notion de «blackness ", véhiculée par le courant hip-hop américain, est instrumentalisée par plusieurs adolescents juifs d'origine éthiopienne dans le but de revendiquer un statut de juif noir dans la société israélienne. Au-delà de l'articulation que ces jeunes trament entre leur expérience de retour et d'insertion en Israël et l'histoire des Afro-Américains dans leur usage de la rhétorique racialisée noir-blanc, le succès commercial du rap, notamment le rappeur gangsta américain Tupac Shakur, ayant vendu plus de 25 millions d'albums, figure au centre de leurs constructions 
narratives. Comme l'indique la popularité du rappeur américain 50 cents à l'échelle mondiale, une autre icône de la marchandisation de la culture de la dénonciation hip-hop, les revendications identitaires des jeunes Israéliens d'origine éthiopienne reposent sur les succès commerciaux plutôt que sur les discours engagés. De surcroît, ces jeunes Israéliens, motivés par leur engagement profond envers le judaïsme et l'idéologie sioniste, cherchent à s'insérer dans la majorité israélienne et non à créer un mouvement de contestation malgré la discrimination qu'ils vivent souvent au quotidien.

En référence au contexte sénégalais, Abdoulaye Niang aborde la question de l'intrication du discours religieux et de la musique rap à travers la figure du «MC prêcheur». Cette figure récente de la culture populaire sénégalaise se situe à la croisée de l'engagement politique et du «business». Au Sénégal, les dilemmes auxquels les acteurs de la culture hip-hop font face se configurent autour de deux enjeux : d'une part, celui de la contradiction apparente entre les normes et les valeurs musulmanes dominantes de la société sénégalaise et les idéaux libertaires et violents du rap commercial nord-américain; et, d'autre part, celui de la professionnalisation du hip-hop dans un contexte où la pratique du hip-hop est perçue de plus en plus comme un moyen d'insertion socioprofessionnelle. Niang démontre que le MC prêcheur est une modalité privilégiée pour faire face à ces dilemmes, arrimant les valeurs dominantes de la société sénégalaise à l'«hypermarché socioculturel du monde» selon ses propres termes.

Dans le cas cubain décrit par Alexandrine Boudreault-Fournier, l'ambiguité se vit plutôt en relation à l'idéologie révolutionnaire, tandis que dans le cas des artistes de la culture hip-hop en France, décrit par Hugues Bazin, Naïm Bornaz et Mehdi Slimani, elle s'articule aux enjeux de la professionnalisation, et de la relation entre l'art populaire et l'art dit légitime. Boudreault-Fournier aborde la question des dilemmes du hip-hop à travers la description de la position ambiguë des éducateurs en arts et des promoteurs naturels, qui constituent deux structures administratives de la gestion des ressources culturelles cubaines révolutionnaires. Ayant pour mandat de former et de promouvoir la culture cubaine révolutionnaire, ces deux groupes de professionnels font face aux contradictions idéologiques entre les politiques du ministère de la Culture cubain dans la Batalla de Ideas (bataille des idées) qui vise le renouveau des diktats de la révolution et l'attrait que représente le reggaeton dans l'industrie commerciale musicale. Inspirée par les perspectives théoriques élaborées par Stuart Hall et Ernesto Laclau, l'auteure montre que face aux contradictions inhérentes à ces deux morales, les 
intervenants de l'État élaborent une articulation idéologique qui arrime les forces dominantes aux forces alternatives et émergentes.

Bazin, Bornaz et Slimani, quant à eux, recadrent la question des dilemmes du hip-hop en termes de la tension entre la culture populaire et l'art dit légitime en France dans le contexte de la professionnalisation progressive des acteurs de la culture hip-hop. La réflexion de ces trois auteurs, dont un chercheur, un rappeur et un chorégraphe, est le fruit d'une collaboration expérimentale de recherche-action qui tente de poser les questions suivantes: "Avec le recul d'un quart de siècle, le hip-hop a-t-il su faire sa marque dans les sociétés contemporaines?» «Le hip-hop a-t-il su garder une originalité et préserver les critères populaires de son émergence?» Le hiphop en France est-il toujours autonome face aux cadres institutionnalisés de la production culturelle? Constitue-t-il toujours une culture de la rue? S'appuie-t-il toujours sur l'innovation comme mode de résolution des problèmes sociaux? Se constitue-t-il encore en tant qu'acteur social révélateur des tensions de la société française en mutation profonde?

Les auteurs concluent que le propre du hip-hop est d'avoir abordé les processus de création, de transmission, de présentation et de diffusion globalement alors que, usuellement en France, ils sont assignés à des fonctions et à des lieux institutionnels distincts. En questionnant la division instituée du «travail de la culture", les auteurs, autant dans leur pratique expérimentale de recherche-action que dans leur regard critique posé sur la culture hip-hop française, replacent le «travail de la culture» au centre des enjeux politiques. Ainsi, le talent des artistes engagés n'exclut pas la possibilité de liens avec le monde commercial ; le ludique et l'aspiration à la reconnaissance professionnelle n'excluent pas non plus l'engagement.

Jenny Mbaye montre comment, depuis le milieu des années 1980, le hip-hop en Afrique de l'Ouest y a significativement encouragé la vitalité du secteur musical tout en impulsant des mouvements sociaux de jeunesse dans le contexte du néolibéralisme économique et de la démocratisation. L'auteure analyse l'engagement et la mobilisation politique, qu'elle qualifie d' " activisme musical», des jeunes artistes du collectif AURA, originaires de dix pays: Bénin, Burkina Faso, Gambie, Guinée, Côte d'Ivoire, Niger, Mali, Mauritanie, Sénégal et Togo. Elle veut montrer comment les acteurs de la culture hip-hop transnationale urbaine, conçue généralement dans la littérature en termes d'enjeux et de dilemmes à l'échelle nationale, se pensent et agissent au-delà des frontières nationales à l'échelle continentale panafricaine. Ainsi la problématique de la marginalisation politico-économique se 
lie à celle de la jeunesse sur le continent, et la culture hip-hop se constitue à l'interstice de ces deux expériences.

Dans le cadre des deux projets pilotés par AURA: la comédie musicale hip-hop «Poto Poto» inaugurée en 2006 et soutenue par une Organisation non gouvernementale $(\mathrm{ONG})$, et la mobilisation autour du single "On ne signe pas», la formation AURA dénonce le statut des jeunes et des autres cadets sociaux en Afrique (les femmes, les pauvres), mais elle le fait aussi en se positionnant en intermédiaire entre les autorités politiques et les populations; l'objectif étant de «synthétiser et de vulgariser le discours politique» (officiel et de résistance) "afin de le rendre accessible à la population dans son ensemble» en espérant «donner les moyens d'agir et de changer leur avenir» aux populations des pays africains. "Prendre le micro» devient une autre manière de «faire de la politique», mais dans le cadre institué de la démocratisation à l'échelle nationale. La résistance et la dénonciation ne se conçoivent donc pas en relation dichotomique avec les figures de l'autorité et du pouvoir politique, mais dans un rapport diffus, oblique même.

Enfin, Myriam Laabidi se penche sur le dilemme que pose la question de la place centrale jouée par le désir d'intégrer l'industrie culturelle locale chez certains acteurs de la culture hip-hop au Québec. Ce souci semble les mettre en porte-à-faux face à la trame historique du hip-hop comme vecteur de revendication et de conscientisation transnationales. À première vue, la surreprésentation des discours de «l'épanouissement personnel au moyen de la création artistique» semble dissociée des préoccupations politiques. Le désintérêt réel exprimé par les artistes hip-hop québécois surtout face à l'univers politique institutionnalisé semble correspondre à une désillusion profonde face au potentiel transformatif de la classe politique locale, surtout en ce qui a trait aux revendications de caractère nationaliste. Mais, plus significatif, l'apparente absence de volonté contestataire s'explique, selon l'auteure, parce que la culture hip-hop québécoise est largement dominée par les francophones de «souche», malgré son caractère plus ethniquement diversifié dans la ville de Montréal.

Toutefois, en raison des politiques identitaires qui entourent la naissance de la culture du hip-hop aux États-Unis, les productions artistiques des Québécois de «souche» doivent être reconnues collectivement par celles des minorités visibles. Laabidi suggère que pour que ce passage puisse avoir lieu, les artistes ont tendance à choisir des thèmes et des messages plus personnels et rétrospectifs qui recoupent les préoccupations plus générales des jeunes et qui sont aussi souvent liées aux enjeux du passage à la vie adulte. Cela 
expliquerait l'apparente neutralité, ou même l'indifférence politique, à des fins d'inclusion culturelle et même de commercialisation.

En conclusion, les contributions de ce numéro spécial mettent en évidence, dans une perspective transculturelle, les diverses postures mises en œuvre par les acteurs de la culture hip-hop face aux nombreux dilemmes qui l'animent; ces derniers se déploient bien au-delà de la dichotomie classiquement évoquée entre la marchandisation et la résistance/dénonciation/ revendication. Du reggaeton portoricain à Cuba à l'activisme musical de AURA en Afrique de l'Ouest, en passant par l'appropriation des discours et des symboles de la négritude transnationale par les jeunes Israéliens d'origine éthiopienne, malgré la force homogénéisatrice des vecteurs économiques de la mondialisation culturelle, les multiples expressions de la culture hip-hop continuent d'être au carrefour des préoccupations quotidiennes de ceux qui $\mathrm{y}$ adhèrent, tout en articulant des formes dénonciatrices, festives et provocatrices. Les narrations autobiographiques et les expressions performatives des multiples expériences de la marginalisation tissent de façon créative des liens avec le politique tel qu'il se définit dans les divers espaces nationaux et continentaux abordés dans ces sept articles.

De fait, aujourd'hui dans plusieurs milieux populaires, le hip-hop est devenu outil de mobilisation sociale, politique et culturelle. En témoignent, entre autres, les divers concerts rap présentés par des jeunes artistes africains qui visent à conscientiser le public aux problèmes de la jeunesse et à encourager la démocratisation des structures de pouvoir, les concerts organisés par des rappeurs israéliens et palestiniens afin de protester contre la situation dans les territoires occupés, les modèles coopératifs du «travail de la culture » proposés par les artistes du hip-hop en France ou encore les MC prêcheurs du Sénégal qui attestent de la créativité de la logique de la débrouille en contexte de précarité économique. 